\title{
NECESSIDADES DE APRENDIZAGEM ACERCA DA CIRURGIA CARDÍACA NA PERSPECTIVA DE PACIENTES E ENFERMEIROS
}

\author{
Learning needs about cardiac surgery from the perspective of patients and nurses
}

Necesidades de aprendizaje acerca de la cirugía cardíaca en la perspectiva de pacientes y enfermeros

\author{
Débora de Almeida Pereira', Tamyres Millena Ferreira², Jadiane Ingrid da Silva², \\ Eduardo Tavares Gomes ${ }^{4 *}$, Simone Maria Muniz da Silva Bezerra ${ }^{5}$
}

RESUMO: Objetivo: Verificar a concordância entre a percepção dos enfermeiros sobre a necessidade de aprendizagem dos pacientes em período pré-operatório acerca da cirurgia cardíaca e os tópicos sobre os quais os pacientes mostram menor conhecimento. Método: Trata-se de um estudo descritivo-exploratório, realizado em duas etapas, em dois hospitais universitários na cidade do Recife, Pernambuco, Brasil. Em uma primeira etapa, os pesquisadores questionaram 30 enfermeiros sobre quais tópicos eles acreditavam que os pacientes tivessem mais dúvidas ou menor conhecimento. Na segunda etapa, 50 pacientes foram entrevistados para verificar seu conhecimento acerca dos 18 tópicos que os enfermeiros haviam julgado. Resultados: A média de acertos dos pacientes foi igual a 5,92. Em 16 dos 18 tópicos avaliados, o conhecimento dos pacientes era menor na perspectiva dos enfermeiros do que nos valores representados pelos acertos dos próprios pacientes. Conclusão: Os tópicos que os pacientes tiveram mais dúvidas devem se tornar focos de atenção no processo educativo do enfermeiro, que necessita conhecer a população sob seus cuidados para melhor planejar suas ações.

Palavras-chave: Educação em saúde. Período pré-operatório. Cirurgia cardíaca. Cuidados de enfermagem.

ABSTRACT: Objective: To verify the agreement between nurses' perceptions about learning needs of patients in the preoperative period of cardiac surgery, and the topics that patients show to have less knowledge about. Method: Descriptive-exploratory study carried out in two steps, at two university hospitals in the city of Recife, Pernambuco, Brazil. In the first step, researchers questioned 30 nurses on which topics they believed patients had more questions or less knowledge about. In the second step, 50 patients were interviewed so as to check their knowledge about the 18 topics that the nurses had judged. Results: The mean of correct answers was 5.92. In 16 of the 18 topics assessed, patients had less knowledge in nurses' perspective than their correct answers represented. Conclusion: The topics patients had more queries about should become the focus of attention in nurses' formation, as these professionals should be acquainted with the population they're caring for in order to better plan their actions.

Keywords: Health education. Preoperative period. Thoracic surgery. Nursing care.

RESUMEN: Objetivo: Verificar la concordancia entre la percepción de los enfermeros sobre la necesidad de aprendizaje de los pacientes en período preoperatorio acerca de la cirugía cardiaca y los tópicos sobre los cuales los pacientes muestran menor conocimiento. Método: Se trata de un estudio descriptivo-exploratorio, realizado en dos etapas, en dos hospitales universitarios en la ciudad de Recife, Pernambuco, Brasil. En una primera etapa, los investigadores cuestionaron a 30 enfermeros sobre qué tópicos creían que los pacientes tenían más dudas o menor conocimiento. En la segunda etapa, 50 pacientes fueron entrevistados para verificar su conocimiento acerca de los 18 tópicos que los enfermeros habían juzgado. Resultados: El promedio de aciertos de los pacientes fue igual a 5,92. En 16 de los 18 tópicos evaluados, el conocimiento de los pacientes era menor en la perspectiva de los enfermeros que en los valores representados por los aciertos de los propios pacientes. Conclusión: Los tópicos que los pacientes tuvieron más dudas deben convertirse en focos de atención en el proceso educativo del enfermero, que necesita conocer a la población bajo sus cuidados para mejor planificar sus acciones. Palabras clave: Educación en salud. Periodo preoperatorio. Cirugía torácica. Atención de enfermería.

'Enfermeira pela Universidade de Pernambuco - Recife (PE), Brasil.

${ }^{2}$ Acadêmica de Enfermagem da Universidade de Pernambuco - Recife (PE), Brasil.

${ }^{3}$ Residente de Enfermagem em Cardiologia do Pronto-Socorro Cardiológico Universitário de Pernambuco Professor Luiz Tavares - Recife (PE), Brasil.

«Mestre em Enfermagem pela Universidade de Pernambuco; Enfermeiro Assistencial da Unidade de Blocos Cirúrgicos do Hospital das Clínicas da Universidade Federal de Pernambuco - Recife (PE), Brasil.

${ }_{5}^{5}$ Pós-Doutora em Enfermagem Fundamental pela Escola de Enfermagem de Ribeirão Preto da Universidade de São Paulo - Ribeirão Preto (SP), Brasil.

*Autor correspondente: edutgs@hotmail.com

Recebido: 30/11/2017 - Aprovado: 04/03/2018

DOI: 10.5327/Z1414-4425201800020005 


\section{INTRODUÇÃO}

O período pré-operatório é destinado ao processo de preparação do paciente em todos os aspectos, além de ser o momento para esclarecer dúvidas quanto aos procedimentos, sobre como ele deve se preparar e como pode ser o pós-operatório ${ }^{1,2}$. A espera de uma cirurgia é vista, muitas vezes, como o risco de incapacidade, morte, ou como a possibilidade de alteração da imagem corporal, o que gera angústia e ansiedade ${ }^{3}$.

A ansiedade e o estresse trazem repercussões psicológicas e fisiológicas que podem desencadear diminuição da capacidade de recuperação dos tecidos e resposta imunológica lenta, contribuindo para uma maior predisposição a infecções ${ }^{4}$.

O enfermeiro, para implementar a educação em saúde, deve buscar compreender as necessidades de aprendizagem dos pacientes e ter as evidências científicas necessárias para organizar o cuidado e orientar o processo educativo 5 . A educação em saúde promove reflexão e consciência crítica, com ênfase em um processo dialógico e organizador da forma de se trabalhar com pessoas, não sendo apenas um ato meramente instrucional ${ }^{6}$. Sendo assim, o enfermeiro deve saber se comunicar com o outro, entender as demandas do paciente e, em sentido mais popular, falar a língua do paciente.

No intuito de tornar a prática da educação em saúde mais efetiva e eficiente no período pré-operatório de cirurgia cardíaca, é importante pensar em estratégias com linguagem acessível ao público-alvo e propor ações educativas e interativas entre o profissional e o paciente. É de suma relevância a realização de orientações e visitas pré-operatórias, visto que elas diminuem a ansiedade e aumentam a adesão à proposta terapêutica ${ }^{2,5,7}$.

\section{OBJETIVO}

Verificar a concordância entre a percepção dos enfermeiros sobre a necessidade de aprendizagem dos pacientes em período pré-operatório acerca da cirurgia cardíaca e os tópicos sobre os quais os pacientes mostram menor conhecimento.

\section{MÉTODO}

Trata-se de um estudo exploratório, descritivo, com abordagem quantitativa.

A pesquisa foi realizada em duas etapas, em dois hospitais universitários na cidade do Recife, Pernambuco, Brasil, sendo um especializado e referência em cardiologia, e outro que atende, além de cirurgia cardíaca, a outras especialidades cirúrgicas.
Em uma primeira etapa, os pesquisadores questionaram 30 enfermeiros sobre quais tópicos eles acreditavam que os pacientes tivessem mais dúvidas ou menor conhecimento. Para se obter tal resposta, foram utilizados os conteúdos que são ministrados na estratégia de educação em saúde do hospital, as dúvidas mais frequentes e a experiência dos autores com o tema. O conteúdo utilizado nas orientações aos pacientes, realizadas no hospital, foi elaborado após ampla revisão da literatura, além de ser baseado na experiência dos profissionais e nos protocolos do hospital.

Para essa etapa, foi elaborado um instrumento em 18 tópicos que permitia que os enfermeiros avaliassem, em uma escala tipo Likert, de 1 a 5 pontos, o nível de dúvida ou o pouco conhecimento que os pacientes com os quais lidam cotidianamente apresentam sobre determinado tópico. A pontuação 1 foi atribuída à menor dúvida, e para cada tópico que o paciente apresentava maior dúvida, foram empregados 5 pontos.

Para a análise desses dados, os tópicos que apresentavam escores 3, 4 e 5 foram considerados relevantes na perspectiva dos enfermeiros, pois retratam, na visão dos profissionais, as maiores necessidades dos pacientes.

Na segunda etapa, foram avaliados 50 pacientes acerca dos mesmos tópicos. Suas respostas foram classificadas da seguinte forma:

- o paciente não sabe sobre o questionado (quando não houve resposta de sua parte ou quando esteve completamente equivocado);

- o paciente sabe parcialmente sobre o questionado (quando não utilizou corretamente os termos ou não soube detalhes, mas soube o principal sobre o cuidado a que o item se refere);

- o paciente sabe sobre o questionado (quando respondeu corretamente, com suas palavras, sobre o principal cuidado relacionado ao item).

Os autores tiveram o cuidado de não entrevistar pacientes que já haviam sido submetidos a qualquer intervenção educativa, de forma a buscar as dúvidas mais primárias, ou seja, que não haviam sido abordadas por nenhum profissional ${ }^{8}$.

Os dados foram analisados utilizando-se o software SPSS 20.0. Foram comparados, ao final, pelo teste do $\chi^{2}$, a diferença entre o percentual de respostas que os pacientes não sabiam e o percentual de importância atribuída pelos enfermeiros a cada item, considerando-se o nível de significância estatística para valor $\mathrm{p}<0,05$.

A pesquisa foi elaborada com base nos preceitos éticos da Resolução n ${ }^{\circ}$ 466 / 2012, do Conselho Nacional de Saúde (CNS), sendo iniciada após aprovação do Comitê de Ética em Pesquisa do Complexo Hospitalar da Universidade de Pernambuco, via Plataforma Brasil, sob número CAAE: 12600113.4.0000.5192. 


\section{RESULTADOS}

Foram convidados a participar 30 enfermeiros, em sua maioria mulheres $(24 / 80,0 \%)$, com tempo médio de formação de 5,5 anos $( \pm 10,43)$ e tempo de experiência aproximado ao de formação $(5,0 \pm 10,78)$. O tempo de experiência variou entre 4 e 28 anos e todos trabalhavam na área assistencial.

Quanto à formação acadêmica, 18 profissionais $(60,0 \%)$ tinham mestrado em Enfermagem e 27 (90,0\%) tinham especialização em Cardiologia, Clínica Cirúrgica ou em áreas correlatas em saúde do adulto; apenas $2(6,77 \%)$ eram doutores. Vinte (66,7\%) tinham artigos publicados em periódicos na área de Enfermagem em Cardiologia; 17 (56,7\%) participaram ou participavam de pesquisas na área; e 23 (76,7\%) tinham experiência assistencial nessa área; o restante $(7 / 23,3 \%)$ tinha experiência em Clínica Cirúrgica, incluindo assistência nos períodos pré e pós-operatório.

A amostra de 50 pacientes foi composta predominantemente por mulheres (27/54,0\%), com até 65 anos (37/74,0\%), provenientes do interior $(25 / 50,0 \%)$, com companheiros, independentemente do vínculo formal de situação conjugal (26/52,0\%). A principal religião referida foi a católica $(25 / 50,0 \%)$ e 22 pacientes $(44,0 \%)$ eram aposentados. A maioria apresentava índice de massa corpórea (IMC) normal (32/64,0\%). A principal comorbidade na amostra foi a hipertensão arterial $(25 / 50,0 \%)$, seguida de diabetes $(13 / 26,0 \%)$; também foram verificados elevados índices de tabagismo (23/56,0\%) e etilismo (23/56,0\%). Uma parcela de $32,0 \%$ (16) dos pacientes já havia sido submetida a uma cirurgia cardíaca anteriormente. A Tabela 1 apresenta a estatística descritiva dos acertos dos pacientes entrevistados.

Comparando-se os tópicos propostos quanto aos pacientes que não souberam responder e as perspectivas dos enfermeiros sobre as dúvidas e o pouco conhecimento dos pacientes, pode-se observar que o percentual de pacientes que não soube realmente responder era menor do que a estimativa dos enfermeiros, considerando-se 16 dos 18 tópicos (Tabela 2). Isso significa que, para os enfermeiros, o conhecimento dos

Tabela 1. Estatísticas descritivas do número de acertos entre os pacientes entrevistados. Recife, Pernambuco, Brasil, 2017.

\begin{tabular}{|l|c|c|c|c|c|c|c|}
\hline & Mediana & Média & Desvio padrão & Mínimo & Máximo & Percentil 25 & Percentil 75 \\
\hline Número de acertos & 5,0 & 5,92 & 4,35 & 1,0 & 15,0 & 4,0 & 8,0 \\
\hline
\end{tabular}

Tabela 2. Comparação dos tópicos quanto aos pacientes que não souberam responder e as perspectivas dos enfermeiros sobre dúvidas e pouco conhecimento dos pacientes. Recife, Pernambuco, Brasil, 2017.

\begin{tabular}{|l|c|c|c|}
\hline Proposição & $\begin{array}{c}\text { Paciente não soube } \\
\text { responder (n/\%) }\end{array}$ & $\begin{array}{c}\text { Perspectiva do } \\
\text { enfermeiro (n/\%) }\end{array}$ & \begin{tabular}{c} 
Valor $\mathbf{p}$ \\
\hline 1. Qual a cirurgia a ser realizada?
\end{tabular} \\
\hline 2. Qual o motivo da realização dessa cirurgia? & $18 / 36,0$ & $26 / 86,7$ & $<0,001$ \\
\hline 3. Como é realizado esse tipo de cirurgia? & $16 / 32,0$ & $29 / 96,7$ & $<0,001$ \\
\hline 4. O que é jejum? & $35 / 70,0$ & $17 / 56,7$ & 0,238 \\
\hline 5. Quanto tempo é necessário fazer jejum antes da cirurgia? & $02 / 4,0$ & $19 / 63,3$ & $<0,001$ \\
\hline 6. Qual o motivo do jejum? & $32 / 64,0$ & $30 / 100,0$ & $<0,001$ \\
\hline 7. Qual tricotomia é necessária? & $40 / 80,0$ & $24 / 84,0$ & 1 \\
\hline 8. Após a cirurgia pode tossir? & $15 / 30,0$ & $23 / 76,7$ & $<0,001$ \\
\hline 9. Qual a posição para dormir no hospital após a cirurgia? & $40 / 80,0$ & $29 / 96,7$ & 0,046 \\
\hline 10. Em que local você estará quando despertar da cirurgia? & $11 / 22,0$ & $27 / 90,0$ & $<0,001$ \\
\hline 11. Como será sua alimentação e dieta após a cirurgia? & $17 / 34,0$ & $28 / 93,3$ & $<0,001$ \\
\hline 12. Há possibilidade de retorno às atividades de vida diária & $25 / 50,0$ & $30 / 100,0$ & $<0,001$ \\
\hline realizadas antes da cirurgia? & $17 / 34,0$ & $28 / 93,3$ & $<0,001$ \\
\hline 13. Há possibilidade de retorno às atividades físicas? & $20 / 40,0$ & $27 / 90,0$ & $<0,001$ \\
\hline 14. Poderá fazer esforço físico desgastante, como pegar & $05 / 10,0$ & $26 / 86,7$ & $<0,001$ \\
\hline objetos pesados após alta? & $24 / 48$ & $30 / 100,0$ & $<0,001$ \\
\hline 15. Há possibilidade de vida sexual normal após a alta? & $36 / 72$ & $30 / 100,0$ & $<0,001$ \\
\hline 16. Quais os cuidados com a ferida operatória pós-alta? & $34 / 68$ & $30 / 100,0$ & $<0,001$ \\
\hline 17. Quais os sinais de infecção da ferida operatória? & $0 / 0$ & $30 / 100,0$ & $<0,001$ \\
\hline 18. Poderá voltar a fumar após a cirurgia? & & & \\
\hline
\end{tabular}


pacientes é menor do que o que eles realmente apresentaram. Apenas no item 6, referente ao motivo do jejum, o percentual foi igual $(80,0 \%)$, e no item 3 , sobre como era realizado o tipo de cirurgia, os enfermeiros estimaram que eles saberiam responder menos do que eles responderam $(\mathrm{p}=0,238)$.

\section{DISCUSSÃO}

É de suma importância a elaboração de escalas e instrumentos validados para avaliar aspectos subjetivos relacionados ao cuidado em saúde e, em particular, ao conhecimento dos pacientes ${ }^{9-11}$. Esses instrumentos possibilitam avaliação mais precisa e proposição mais focada e otimizada de medidas de intervenção, com o propósito de prestar assistência de melhor qualidade a esses pacientes ${ }^{11,12}$.

As ações educativas do enfermeiro para pacientes e família, além de reduzirem a ansiedade, aumentam a adesão ao autocuidado, necessário tanto no pré quanto no pós-operatório ${ }^{13}$. Estudos mostram que a adesão ao processo de reabilitação está associada ao fato de o paciente ter consciência a respeito do procedimento pelo qual será submetido e seu processo de recuperação ${ }^{14}$. As intervenções de Enfermagem no período pré-operatório são fundamentais para que o paciente se empodere do conhecimento necessário para se tornar responsável, com a equipe, pelo seu processo de recuperação e autocuidado ${ }^{15}$.

Dos resultados, pode-se inferir que os enfermeiros consideravam o conhecimento dos pacientes menor do que o referido por estes ao acertarem as questões, mesmo que parcialmente. Contudo, o fato de os enfermeiros subestimarem o conhecimento dos pacientes não repercute diretamente no processo de educação, visto que o contrário seria preocupante.

Erros sobre aspectos que podem ser considerados básicos, tais como o tipo de cirurgia e o motivo (40; 80,0\%) e o tempo de jejum (32; 64,0\%), representam pouco conhecimento, que pode ser minimizado pela visita pré-operatória, a ser realizada pelo enfermeiro do setor ou mesmo pelo profissional do Centro Cirúrgico. Contudo, o enfermeiro do período pré-operatório pode dispor de outras estratégias para educação em saúde, como confecção de cartilhas, vídeos educativos e palestras expositivas. Ressalta-se que questões acerca do pós-operatório também devem ser enfatizadas, considerando que há evidência de que isso contribui para a ansiedade pré-operatória, a recuperação e a adaptação do paciente, inclusive do tempo de permanência na terapia intensiva e da incidência de complicações pós-operatórias ${ }^{16-18}$. Na amostra, questões sobre autocuidado no pós-operatório não tiveram resultados satisfatórios.
De forma geral, pode-se afirmar que a média de acertos $5,92( \pm 4,35)$ foi baixa, considerando que o percentil de $75,0 \%$ dos pacientes acertou até 8 questões, de um total de 18, ou seja, menos da metade (Tabela 1). Considerando que a distribuição das respostas corretas teve de 1 até 15 tópicos respondidos corretamente, deve-se continuar investigando fatores que possam acarretar melhorias nos resultados, inclusive o acesso à internet, os meios de comunicação, a presença de acompanhantes.

Uma pesquisa nacional sobre validação clínica do Diagnóstico de Enfermagem "Conhecimento deficiente" apontou que mais de $75,0 \%$ dos pacientes o apresentavam, similar ao achado em outros trabalhos ${ }^{19-21}$. Esse percentual é próximo da perspectiva dos enfermeiros do presente estudo acerca do pouco conhecimento dos pacientes sobre a cirurgia cardíaca.

Outra pesquisa, com 80 pacientes, utilizando-se de um questionário específico sobre doença coronária e revascularização, encontrou que mais de $50,0 \%$ dos pacientes que compuseram a amostra erraram ou não souberam responder às questões referentes ao nome da doença, aos sinais e sintomas de complicação, aos objetivos e ao tipo de cirurgia e anestesia ${ }^{22}$. Ainda não há, no Brasil, questionário que aborde o conhecimento dos pacientes em pré-operatório acerca de cirurgias cardíacas.

A pesquisa apresentada teve por limitação não investigar se os pacientes haviam buscado alguma informação anterior sobre a cirurgia que pudesse impactar, de forma significativa, no seu conhecimento, como, por exemplo, acesso à internet, experiência de amigos, parentes e até colegas de enfermaria que realizaram a cirurgia anteriormente.

\section{CONCLUSÃO}

Os enfermeiros mostraram que consideravam o conhecimento dos pacientes menor do que o realmente apresentado por estes ao acertarem as questões, mesmo que parcialmente. Entre os 18 tópicos avaliados, em 16 deles o conhecimento dos pacientes era menor na perspectiva dos enfermeiros do que nos valores representados pelos acertos dos próprios pacientes.

Os tópicos que os pacientes tiveram mais dúvidas devem se tornar foco de maior atenção no processo educativo do enfermeiro.

Para dar continuidade a este estudo, os autores seguirão na elaboração e validação de uma escala para avaliar o conhecimento de pacientes no período pré-operatório acerca da cirurgia cardíaca. Com essa escala, enfermeiros assistenciais poderão verificar na população sob seus cuidados quais tópicos suscitam mais dúvidas, bem como avaliar o impacto das estratégias educativas utilizadas. 


\section{REFERÊNCIAS}

1. Costa TMN, Sampaio CEP. As orientações de enfermagem e sua influência nos níveis de ansiedade dos pacientes cirúrgicos hospitalares. Rev Enferm UERJ. 2015;23(2):260-5. http://dx.doi.org/10.12957/reuerj.2015.16534

2. Teixeira MV, Corrêa AR, Silqueira SMF, Carvalho DV. Avaliação dos resultados das orientações pré-operatórias a pacientes submetidos à cirurgia cardíaca eletiva. Rev Enferm Cent 0 Min. 2013;3(2):62031. http://dx.doi.org/10.19175/recom.v0i0.329

3. Erdmann AL, Lanzoni GMM, Callegaro GD, Baggio MP, Koerich C. Compreendendo o processo de viver significado por pacientes submetidos à cirurgia de revascularização do miocárdio. Rev Latinoam Enferm. 2013;21(1):[08 telas].

4. Veiga EP, Gomes L, Melo GF. Fatores estressores em Unidade de Terapia Intensiva: percepção de pacientes idosos e adultos no pósoperatório de cirurgia cardíaca. Kairós Gerontol. 2013;16(3):65-77.

5. Ribeiro CP, Silveira CO, Benetti ERR, Gomes JS, Stumm SEM. Diagnósticos de enfermagem em pacientes no pós-operatório de cirurgia cardíaca. Rev RENE. 2015;16(2):159-67. http://dx.doi. org/10.15253/rev\%20rene.v16i2.2697

6. Sales PC, Bezerra SMMS. Educação popular como estratégia para a promoção da saúde cardiovascular brasileira: uma revisão integrativa. Saúde Colet. 2013;10(59):55-9.

7. Schmitz CR, Klock P, Santos JLG, Erdmann AL. Orientações no préoperatório de cirurgia cardíaca a pacientes idosos: revisão integrativa. Rev EnfermUERJ. 2013;21(3):391-6.https://doi.org/10.12957/reuerj.2013.7549

8. Pereira DF, Ferreira TM, Gomes ET, Silva T, Bezerra SMMS. Conhecimento de pacientes no pré-operatório acerca da cirurgia cardíaca. Rev Enferm UFPE online. 2017;11(Suppl. 6):2557-64.

9. Pasquali L. Psicometria: teoria dos testes na psicologia e na educação. 3a ed. Petrópolis: Vozes; 2009.

10. Tibúrcio MP, Melo GSM, Balduíno LSC, Costa IKF, Dias TYAF, Torres GV. Validation of an instrument for assessing the ability of blood pressure measurement. Rev Bras Enferm. 2014;67(4):581-7. http:// dx.doi.org/10.1590/0034-7167.2014670413

11. Bonin CDB, Santos RZ, Ghisi GLM, Vieira AM, Amboni R, Benetti M. Construção e validação do questionário de conhecimentos para pacientes com insuficiência cardíaca. Arq Bras Cardiol. 2014;102(4):36473. http://dx.doi.org/10.5935/abc.20140032

12. Fortunato JGS, Furtado MS, Hirabae LFA, Oliveira JA. Escalas de dor no paciente crítico: uma revisão integrativa. Rev HUPE. 2013;12(3):1107. DOI: $10.12957 /$ rhupe. 2013.7538
13. Silveira LL, Almeida MA, Silva MB, Nomura ATG. Registros de enfermagem acerca das ações educativas para pacientes submetidos à artroplastia de quadril. Rev Eletr Enf. 2015;17(4). https://doi. org/10.5216/ree.v17i4.31636

14. Camponogara S, Soares SGA, Silveira M, Viero CM, Barros CS, Cielo C. Percepção de pacientes sobre o período pré-operatório de cirurgia cardíaca. REME Rev Min Enferm. 2012;16(3):382-90. http://www. dx.doi.org/S1415-27622012000300010

15. Ong J, Miller PS, Appleby R, Allegretto R, Gawlinski A. Effect of a preoperative instructional digital video disc on patient knowledge and preparedness for engaging in postoperative care activities. Nurs Clin North Am. 2009;44(1):103-15. https://doi.org/10.1016/j. cnur.2008.10.014

16. Felippe CM, Roque LO, Ribeiro IM. Contribuições das orientações pré-operatórias na recuperação de pacientes submetidos a cirurgias cardíacas. Rev Pesq Saúde. 2013;14(3):160-5.

17. Guo R, East L, Arthur A. A preoperative education intervention to reduce anxiety and improve recovery among Chinese cardiac patients: a randomized controlled trial. Int J Nurs Stud. 2012;49:129-37. https:// doi.org/10.1016/j.ijnurstu.2011.08.008

18. Kalogianni A, Almpani P, Vastardis L, Baltopoulos G, Charitos C, Brokalaki H. Can nurse-led preoperative education reduce anxiety and postoperative complications of patients undergoing cardiac surgery? Eur J Cardiovasc Nurs. 2016;15(6):447-58. https://doi. org/10.1177/1474515115602678

19. Lopes CT, Carneiro CS, Santos VB, Barros ALBL. Diagnósticos de Enfermagem validados em cardiologia no Brasil: revisão integrativa de literatura. Acta Paul Enferm. 2012; 25(n. esp. 1):155-60. http:// dx.doi.org/10.1590/S0103-21002012000800024

20. Galdeano LE, Rossi LA, Pelegrino FM. Validação de conteúdo do diagnóstico de enfermagem conhecimento deficiente. Acta Paul Enferm. 2008;21(4):549-55.

21. Fernandes CM, Rangel N, Paula AC, Fernandes E, Silva RA, Reis S, et al. Nursing diagnoses identified in pre-surgical of cardiac surgery patients. Rev Enferm UFPE online. 2010;4(esp):1092-100. http:// dx.doi.org/10.5205/reuol.958-8027-5-LE.0403esp201022

22. Galdeano LE, Rossi LA, Dantas RAS. Deficient knowledge nursing diagnosis: identifying the learning needs of patients with cardiac disease. Int J Nurs Terminol Classif. 2010;21(3):100-7. https://doi. org/10.1111/j.1744-618X.2010.01155.x 